Produto \& Produção, vol. 6, n. 2, p. 01-11, jun. 2002

\title{
Modelo acumulativo de prioridades competitivas: validação teórica e impacto em performance
}

\author{
Giovani José Caetano da Silveira \\ University of Calgaray/CA \\ Flávio S. Fogliatto \\ PPGEP/UFRGS
}

Este artigo apresenta uma análise empírica do modelo acumulativo de prioridades competitivas, originalmente proposto por Nakane (1986). O modelo provê uma seqüência para o desenvolvimento de distintas prioridades competitivas em operações, tal que melhorias em prioridades anteriores possam auxiliar no desenvolvimento de prioridades posteriores. Esta é uma alternativa à visão de trade-offs estáticos entre prioridades competitivas. Testamos o modelo acumulativo analisando dados da edição de 1996 do International Manufacturing Strategy Survey (IMSS-II). Um conjunto de 283 plantas de manufatura de produtos metal-mecânicos, maquinário e equipamentos provenientes de 23 países foi analisado. Realizamos uma análise estatística multivariada nos dados, para testar três postulados básicos do modelo acumulativo: (i) a seqüência sugerida para o desenvolvimento de prioridades competitivas (testamos a seqüência proposta por Noble, 1995), (ii) qualidade como a primeira prioridade competitiva a ser desenvolvida, e (iii) o impacto positivo do modelo sobre o desempenho econômico da firma.

Palavras chave: prioridades competitivas; modelo acumulativo; modelo do cone de areia; estratégia de produção; análise multivariada.

This paper searches for empirical evidence to the cumulative capability model in Nakane (1986). The model provides a path for the development of a series of operations capabilities, so that improvements in preceding capabilities would support, rather than prevent, improvements in succeeding capabilities. The model may be viewed as a counterpoint to the concept of static trade-offs between competitive priorities. We tested the cumulative capability model through multivariate statistical analysis on data from the 1996 edition of the International Manufacturing Strategy Survey. Data gathered from a group of 283 manufacturers of metal products, machinery, and equipment from 23 countries was analyzed. The objective is to test the validity of three main postulates of the model, that are (i) the proposed sequence for capability developments, (ii) quality as the most basic competitive capability and (iii) the positive relationship between model adoption and business performance improvements.

Keywords: competitive capabilities; cumulative model; sand cone model.

\section{Introdução}

Ao longo das últimas décadas, a competitividade em operações tem sido traduzida em um número crescente de prioridades competitivas. Atualmente, sugere-se que empresas de manufatura e serviços devam desenvolver habilidades superiores não somente em aspectos básicos como custo, qualidade e flexibilidade, mas também em variáveis mais amplas de um sistema de operações, tais como velocidade e pontualidade de entregas, serviços agregados ao consumidor e inovação contínua de produtos e processos. Em vez de focalizarem-se em algumas prioridades competitivas chave, as estratégias de operações devem prover o desenvolvimento de um conjunto amplo de variáveis. Logo, um dos principais desafios para as empre- sas no novo século será o de gerenciar uma variedade crescente de iniciativas de melhoria e variáveis de desempenho, sem serem desnorteadas por mudanças organizacionais constantes.

O modelo acumulativo de prioridades competitivas é uma das principais tentativas de responder a este desafio. Este modelo foi proposto por Nakane (1986) e aprimorado por uma série de estudos baseados no Manufacturing Futures Survey (MFS), especialmente Ferdows e De Meyer (1990) e Roth e Miller (1992). O modelo provê uma seqüência para o desenvolvimento de uma série de prioridades competitivas em operações, tal que melhorias em prioridades antecedentes possam auxiliar, e não limitar, melhorias nas prioridades seguintes. 
Até o momento, o principal debate relativo a este modelo tem focalizado em sua validação empírica. Com exceção de Noble (1995), essas análises sempre utilizaram dados do mesmo estudo (MFS) que originou o modelo, apesar de basearem-se em diferentes edições e países de origem. Enquanto Roth e Miller (1992) e De Meyer (1998) encontraram uma evidência positiva para o modelo, Ferdows e De Meyer (1990) não puderam corroborar a seqüência por eles proposta. Noble (1995) encontrou evidência parcial para o modelo na base coreana de dados provenientes de um outro estudo, enquanto os resultados das bases norte-americana e européia foram pouco conclusivos. Logo, ainda há necessidade de testar-se, formalmente, o modelo acumulativo, especialmente utilizando dados de estudos alternativos e com uma maior variedade de países de origem.

Como resposta a este problema, este artigo busca validar o modelo acumulativo de prioridades competitivas através da análise estatística dos dados da edição de 1996 do International Manufacturing Strategy Survey (IMSS-II). O objetivo é testar três postulados básicos do modelo acumulativo, que são (i) a seqüência sugerida para o desenvolvimento de prioridades competitivas (testamos, aqui, a seqüência proposta por Noble, 1995), (ii) qualidade como a primeira prioridade competitiva a ser desenvolvida, e (iii) o impacto positivo do modelo sobre o desempenho econômico das empresas. No que se refere a (i), em particular, este estudo é o primeiro a testar a validade de uma seqüência de desenvolvimento de prioridades competitivas através de análise estatística formal.

\section{0 modelo acumulativo}

Nakane (1986) propôs o modelo acumulativo de prioridades competitivas para descrever as práticas de empresas de manufatura japonesas, que pareciam obter vantagens sobre seus competidores em um grande número de variáveis, simultaneamente. Seu modelo propôs uma seqüência para o desenvolvimento de prioridades competitivas, a saber: qualidade, pontualidade, custo e flexibilidade (uma seqüência similar foi proposta, simultaneamente, por Hall, 1987). O principal pressuposto era que o prévio desenvolvimento de prioridades na base do modelo seria a única maneira de garantir e preservar melhorias obtidas em prioridades próximas ao topo. Este enfoque era oposto ao conceito de trade-offs estáticos em operações (Skinner, 1974, entre outros); tal conceito sugere que sistemas de operações devem focalizar-se em um conjunto limitado de prioridades competitivas.

Estudos posteriores aprimoraram o modelo acumulativo. Ferdows e De Meyer (1990) propuse- ram uma seqüência um pouco diferente, à qual eles denominaram modelo do cone de areia, tendo qualidade e pontualidade como primeira e segunda camadas, seguidas por flexibilidade (velocidade de resposta) e custo. Como no modelo de Nakane, o modelo do cone de areia foi baseado nos resultados do MFS. Além disto, este novo modelo buscou atender a alguns novos pressupostos teóricos; por exemplo, custo como a conseqüência última de melhorias consistentes em outras áreas das operações. Mais tarde, Roth e Miller (1992) sugeriram uma nova seqüência: qualidade, entregas, escopo de mercado (uma prioridade distinta, relacionada à "interface de um sistema de manufatura com seus consumidores e mercados”), flexibilidade e custo. Finalmente, Noble (1995) testou uma versão ampliada do modelo de Nakane, também começando por qualidade e pontualidade, mas seguindo com velocidade de entregas, custo, flexibilidade e inovação.

Apesar de seu grande apelo e aparente lógica, o modelo acumulativo tem sido alvo de constantes críticas, especialmente em relação à sua validação empírica limitada e rigidez. Apesar de sugerirem seqüências para o desenvolvimento de prioridades competitivas, nenhum dos estudos mencionados tiveram sucesso em sua validação. De modo geral, estes estudos restringiram-se a validar a possibilidade de desenvolvimento conjunto de duas ou mais prioridades em uma mesma organização, o que não é o mesmo que testar uma seqüência de desenvolvimento. Com relação ao problema da rigidez, mesmo Ferdows e De Meyer (1990) reconheceram que o modelo por eles proposto não era suficientemente contingente: somente empresas operando notoriamente abaixo dos níveis de qualificação em seu setor deveriam desenvolver prioridades mais problemáticas em primeiro lugar. Ainda assim, os autores consideraram esta rigidez como um aspecto positivo de um modelo que colocava "os princípios fundamentais da boa gerência de manufatura em prática”. Collins e Schmenner (1993) e White (1996) buscaram resolver este problema propondo modelos de múltiplas prioridades, que não fixavam seqüências rígidas de desenvolvimento.

\section{Hipóteses da pesquisa}

Diversos estudos analisaram dados provenientes do MFS na tentativa de obter evidência empírica para o modelo acumulativo de prioridades competitivas. Ferdows e De Meyer (1990) foram os primeiros a trabalhar no sentido da validação do modelo, concluindo que dados correspondentes à porção européia do MFS, obtidos em 1988, não corroboravam a seqüência de prioridades proposta no modelo. Por outro lado, a análise em Roth e Miller (1992), realizada 
sobre a porção norte-americana do MFS, com dados coletados em 1988, apontou para a existência de uma seqüência de prioridades: qualidade, entregas, escopo de mercado, flexibilidade e custo. O estudo em Roth e Miller (1992) teve por objetivo detectar diferenças existentes entre líderes (empresas que atingem plenamente suas metas comerciais) e seguidores (empresas que atingem apenas parte de suas metas comerciais) relativamente a cada prioridade; como esperado, as empresas líderes obtiveram pontuação significativamente maior em todas as prioridades. Em um estudo mais recente, De Meyer (1998) constatou que, num período compreendido entre 1986 e 1996, empresas européias de manufatura enfatizaram pontualidade, flexibilidade e custo, em detrimento de qualidade, como prioridades. Estas constatações, todavia, estão em desacordo com os resultados em Kim e Kim (1999). A análise da porção coreana do MFS feitas por estes autores, sugere que empresas coreanas de manufatura, de acordo com os dados coletados em 1996, atribuíram menor importância a custo (preço baixo) e flexibilidade (alterações nos projetos), se comparados com os resultados compilados em 1988; tal constatação parece desafiar a seqüência de prioridades proposta no modelo acumulativo.

Até a presente data, os estudos de Noble (1995; 1997) foram os únicos a testar o modelo acumulativo usando dados alternativos, não oriundos do MFS. O modelo testado pela autora é uma versão ampliada do modelo em Nakane (1986), incluindo as prioridades qualidade, pontualidade, velocidade nas entregas, custo, flexibilidade e inovação. As descobertas reportadas não são conclusivas, além de oporem-se à grande parte do estudos realizados por outros autores. Enquanto empresas coreanas de manufatura parecem conformar-se à estrutura do modelo acumulativo, empresas norte-americanas e européias apresentaram relações positivas fracas entre prioridades nos níveis mais baixos do modelo. Mais importante ainda, Noble encontrou relações negativas entre as prioridades velocidade nas entregas e flexibilidade, e entre velocidade nas entregas e inovação, o que parece estar mais de acordo com o modelo de tradeoffs do que com o modelo acumulativo. É importante observar que as análises em Noble (1995; 1997) tinham por objetivo validar algum modelo acumulativo de prioridades, e não uma dada seqüência dentro do modelo. Assim, permanece em aberto a verificação empírica do modelo acumulativo a partir de surveys alternativos. Com isto em mente, nós analisamos, neste artigo, a base de dados do IMSS em busca de evidência empírica para a seqüência proposta por Noble, o que resulta na primeira hipótese a ser verificada nesta pesquisa:
H1: Dados do survey vão proporcionar evidência empírica acerca da existência de um modelo acumulativo de prioridades competitivas com sequência de prioridades assim definida: qualidade, pontualidade, rapidez nas entregas, custo, flexibilidade e inovação.

Apesar da dificuldade em validar o modelo acumulativo na íntegra, pode-se encontrar evidência empírica acerca de um de seus postulados, que coloca a prioridade qualidade como básica para melhorias duradoras no desempenho de empresas. Análises feitas com dados do MFS coletados entre 1983 a 1990 (Ferdows et al., 1986; Ferdows e De Meyer, 1988; De Meyer et al., 1989; De Meyer e Ferdows, 1991) sugerem que produzir itens que atendam a altos padrões de qualidade era uma das preocupações centrais de empresas de manufatura na Europa, América do Norte e Japão. Além disso, as análises mostraram que o desenvolvimento da competência em qualidade permitiu às empresas japonesas superar o trade-off entre flexibilidade e custo em tempo hábil. Analisando dados oriundos da porção coreana do MFS, Kim e Kim (1999) identificaram qualidade como a principal prioridade competitiva em empresas coreanas durante três edições consecutivas do survey (1988, 1990 e 1996). Pelo exposto acima, parece intuitivo que a prioridade qualidade deveria relacionar-se positivamente com as demais prioridades do modelo acumulativo. A meta-análise de estudos sobre prioridades em manufatura elaborada por White (1996) associou a prioridade qualidade a melhorias em velocidade nas entregas, flexibilidade e custo. Analogamente, Flynn et al. (1999) descobriram que focalizar na redução da variabilidade de processos resultava em melhoria na pontualidade. Dentre os autores pesquisados neste trabalho, Collins et al. (1998) são os únicos a sugerir qualidade como um fator não influente para obtenção de melhorias em flexibilidade (no referido estudo, flexibilidade é apresentada como um construto ampliado envolvendo fatores como gestão de estoques, introdução de novos produtos e tempo demandado para entregas). Desta forma, nossa análise deveria apontar para qualidade como sendo a principal priorididade competitiva em empresas de manufatura, além de relacionar tal ênfase a uma maior competitividade nas demais prioridades do modelo acumulativo; isto nos leva à segunda e terceira hipóteses de trabalho:

H2: Empresas de manufatura identificam a prioridade qualidade como de maior importância competitiva dentre as prioridades no modelo.

H3: Qualidade como prioridade competitiva relaciona-se positivamente com as demais prioridades competitivas. 
O terceiro principal elemento motivador de análises empíricas sobre o modelo acumulativo é o impacto do desenvolvimento de prioridades sobre o desempenho do negócio. Roth e Miller (1992) associaram diversas estratégias que exploram prioridades do modelo acumulativo a ganhos de lucro e retorno sobre ativos em empresas. Noble (1995; 1997) verificou que empresas que competiam explorando múltiplas prioridades exibiam maiores incrementos de produtividade. De maneira análoga, Spina et al. (1996) determinaram uma relação positiva entre a adoção de uma estratégia de prioridades múltiplas e o grau de melhoria no desempenho de empresas, usando a mesma base de dados (IMSS) utilizada neste trabalho. Em contra-partida, Schmenner e Swink (1998) afirmam que os efeitos sinergísticos preditos pelo modelo acumulativo tenderiam a diminuir à medida que uma planta de manufatura atingisse sua "fronteira” de ativos (desempenho máximo possível de obtenção com ativos disponíveis). Todavia, é importante observar que nenhum dos autores acima testaram a relação entre a adoção de uma determinada sequência de desenvolvimento de prioridades por uma empresa e melhorias no desempenho do negócio. Assim, definimos a hipótese H4 de forma a investigar o impacto da adoção da sequência de prioridades do modelo acumulativo sobre o desempenho de empresas de manufatura:

H4: Foco no desenvolvimento de prioridades seguindo a sequência proposta no modelo de prioridades acumuladas leva a incrementos maiores no desempenho do negócio.

\section{Método de pesquisa}

O presente estudo utiliza dados obtidos da segunda rodada do International Manufacturing Strategy Survey (IMSS-II). O referido survey tem o formato de um questionário dividido em quatro seções: (i) estratégias, objetivos e custos, (ii) práticas atuais de manufatura e integração, (iii) atividades planejadas e atividades já realizadas na manufatura, e (iv) desempenho da manufatura. O IMSS-II foi conduzido durante o ano de 1996, envolvendo 700 empresas em 26 países. Pesquisadores em universidades nos diversos países conduziram o survey. Os questionários preenchidos foram recebidos via postal e agrupados, mais tarde, em um único banco de dados. O presente estudo analisa um subgrupo de 283 empresas respondentes, oriundas de 23 países distintos. Todas as empresas selecionadas (i) retornaram questionários completos à base de dados e (ii) encontram-se cadastradas na divisão 38 do ISIC como fabricantes de produtos metal-mecânicos, maquinário e equipamentos. A estratificação da amostra por país de procedência vem apresentada na Tabela 1. Nosso estudo utiliza dados relacionados às prioridades competitivas e desempenho comercial das empresas respondentes do survey. Os procedimentos de medição das variáveis investigadas são detalhados a seguir.

Prioridades Competitivas - as empresas amostradas foram solicitadas a avaliarem o grau de importância dado a nove objetivos, listados na Tabela 2; as respostas foram motivadas pela pergunta apresentada no cabeçalho da tabela. As avaliações foram medidas utilizando uma escala Likert com 5 pontos e duas âncoras ("nada importante” e “muito importante”). Avaliações dos 283 respondentes foram processadas, totalizando 2547 pontos amostrais.

Desempenho Comercial - melhorias em quatro indicadores de desempenho econômico foram medidas: (a) fatia de mercado (\% de mudança na fatia de mercado da empresa entre 1993 e 1996), (b) lucratividade (\% de mudança na lucratividade entre 1993 e 1996), (c) custo do produto (\% de mudança no custo médio unitário de manufatura entre 1993 e 1996), e (d) retorno sobre investimento (\% de mudança no retorno sobre investimento entre $1993 \mathrm{e}$ 1996). A análise de melhorias no desempenho, ao

Tabela 1 - Estratificação dos dados analisados neste trabalho por país de procedência

\begin{tabular}{|lc|lc|lc|}
\hline Americas & $\boldsymbol{N}$ & Europa & $\boldsymbol{N}$ & Ásia e Pacífico & $\boldsymbol{N}$ \\
\hline Argentina & 12 & Dinamarca & 8 & Austrália & 20 \\
Brasil & 10 & Finlândia & 7 & Hong Kong & 3 \\
Canadá & 19 & França & 1 & Japão & 12 \\
Chile & 7 & Alemanha & 21 & Nova Zelândia & 12 \\
México & 3 & Hungria & 15 & Coréia do Sul & 17 \\
Peru & 6 & Itália & 38 & & \\
Estados Unidos & 18 & Países Baixos & 13 & & \\
& & Noruega & 4 & & \\
\hline
\end{tabular}


invés da análise dos níveis de desempenho, permitiunos visualizar o elemento temporal, necessário para verificação do modelo acumulativo, seguindo a abordagem longitudinal recomendada por White (1996).

\section{Análise dos dados e resultados}

Os objetivos listados na Tabela 2 foram tratados como variáveis aleatórias, cada variável consistindo de 283 realizações. Nosso primeiro objetivo é usar a matriz de correlações $\boldsymbol{\Sigma}$, obtida a partir das variáveis, para identificar similaridades entre elas. Para tanto, uma Análise Fatorial foi conduzida (ver Seber, 1984, entre outros). Os fatores foram obtidos dos autovetores de $\boldsymbol{\Sigma}$ (ou seja, utilizamos extração por componentes principais) e rotados ortogonalmente para melhor ajuste, utilizando uma rotação Varimax.

De um total de nove fatores, cinco foram retidos usando o critério do autovalor unitário (isto é, um fator é retido na análise se reproduzir, no mínimo, a variância de uma variável individual; observe que a determinação dos fatores a partir de $\boldsymbol{\Sigma}$ implica em variáveis com variâncias unitárias). Os cinco fatores extraídos de $\boldsymbol{\Sigma}$ e a variância total explicada por eles estão apresentados na Tabela 3.

Cada fator na Tabela 3 captura uma direção de variabilidade do conjunto de dados originais, que contém as nove variáveis consideradas na análise. Por serem definidos a partir da estrutura de correlações das variáveis, os fatores promovem um agrupamento de variáveis por similaridade. Os fatores são combinações lineares das variáveis. Todas as variáveis encontram-se representadas nos fatores, mas somente algumas são relevantes em sua composição. Para determinar variáveis relevantes em cada fator, anali- sam-se os números (cargas) associados a cada variável no fator em questão. Cargas representam correlações entre variáveis e o fator em questão; assim, quanto maior a carga de uma variável, maior a sua pertinência no fator. Usualmente, um valor de carga igual ou superior a 0,5 define a pertinência (ou relevância) de uma variável em um dado fator (Rencher, 1995; Seber, 1984). Na Tabela 3, cargas $\geq$ 0,5 estão apresentadas na diagonal principal em negrito para melhor visualização da composição dos fatores (os fatores foram ordenados de forma a refletir este padrão de apresentação).

Neste trabalho, fatores e suas respectivas cargas foram utilizados na definição do agrupamento de objetivos. Por exemplo, o agrupamento de variáveis (objetivos) representado pelo Fator 1 reuniu um único objetivo, PVB, com carga dada por 0,975; conseqüentemente, o agrupamento foi denominado Custo. Em contrapartida, o agrupamento de objetivos no Fator 3 reuniu PPQ e MQM, com cargas 0,836 e 0,818 , respectivamente; assim, o agrupamento foi denominado Qualidade, dada a natureza dos objetivos nele reunidos. Os demais agrupamentos foram definidos de maneira similar.

Na Tabela 3, é importante observar que nenhum objetivo pertence a mais de um fator (isto é, apresenta carga $\geq 0,5$ em mais de um fator); esse aspecto possibilitou uma definição precisa dos construtos a partir da análise fatorial. Tal definição dos construtos de prioridade difere daquela apresentada em Noble (1995), resultante de agrupamentos qualitativos de objetivos (ou seja, agrupamentos definidos pelo analista).

Na nossa abordagem, construtos de capacidade foram definidos a partir da estrutura de correlação dos objetivos; somente a denominação dos construtos foi

Tabela 2 - Objetivos listados no questionário e avaliados pelas empresas amostradas

\begin{tabular}{|l|}
\hline Considere o grau de importância dos seguintes objetivos, sob o ponto \\
de vista de seus principais clientes. Em comparação com a \\
competição, você teria a preferência de seus clientes quanto a \\
colocação de pedidos se direcionasse seus esforços na oferta de: \\
\hline Preços de venda mais baixos, PVB \\
\hline Entregas mais rápidas, ER \\
\hline Melhor atendimento a clientes (pós-vendas e/ou apoio técnico), MAC \\
\hline Melhor projeto de produto e qualidade, PPQ \\
\hline Maior qualidade na manufatura, MQM \\
\hline Maior pontualidade nas entregas, MPE \\
\hline Maior flexibilidade no tamanho dos pedidos, FTP \\
\hline Maior variedade de produtos, MVP \\
\hline Maior número de produtos novos, NPN \\
\hline
\end{tabular}


Tabela 3 - Resultados da Análise Fatorial

\begin{tabular}{|lccccc|}
\hline Objetivos $\backslash$ Fatores & Fator $\mathbf{1}$ & Fator $\mathbf{2}$ & Fator $\mathbf{3}$ & Fator $\mathbf{4}$ & Fator 5 \\
\hline Rótulo do fator & Custo & Entrega & Qualidade & Pontualidade & Flexibilidade \\
\hline \hline Preço de venda, PVB & $\mathbf{0 , 9 7 5}$ & 0,004 & $-0,004$ & 0,086 & 0,019 \\
Entrega rápida, ER & 0,220 & $\mathbf{0 , 7 0 3}$ & 0,021 & 0,361 & 0,075 \\
Atendimento a clientes, MAC & $-0,183$ & $\mathbf{0 , 8 2 7}$ & 0,176 & $-0,109$ & 0,127 \\
Projeto de produto, PPQ & $-0,035$ & 0,135 & $\mathbf{0 , 8 3 6}$ & $-0,102$ & 0,155 \\
Qualidade na manufatura, MQM & 0,029 & 0,083 & $\mathbf{0 , 8 1 8}$ & 0,251 & 0,010 \\
Pontualidade nas entregas, MPE & 0,135 & 0,433 & 0,237 & $\mathbf{0 , 5 4 8}$ & 0,033 \\
Tamanho dos pedidos, FTP & 0,035 & 0,001 & 0,013 & $\mathbf{0 , 8 8 8}$ & 0,119 \\
Variedade de produtos, MVP & 0,056 & 0,101 & $-0,037$ & 0,092 & $\mathbf{0 , 8 7 1}$ \\
Produtos novos, NPN & $-0,035$ & 0,070 & 0,214 & 0,057 & $\mathbf{0 , 8 4 5}$ \\
\hline \% da variância explicada & 8,73 & 13,64 & 15,01 & 10,66 & 27,79 \\
Variância acumulada \% & 8,73 & 22,37 & 37,38 & 48,04 & 75,83 \\
Alfa de Cronbach & 0,65 & 0,57 & 0,60 & 0,64 & 0,78 \\
\hline
\end{tabular}

determinada subjetivamente. Essa denominação é consistente com a interpretação realizada em trabalhos anteriores que igualmente definiram construtos de prioridades estratégicas a partir de análise fatorial de variáveis de objetivos. Por exemplo, Ward et al. (1998) similarmente denominam Entrega (Delivery) ao fator envolvendo "entrega rápida" (short delivery time), além de Flexibilidade (Flexibility) ao fator envolvendo "variedade de produtos" (large number of product features or options) e "produtos novos" (new products into production quickly). Porth et al. (1998) igualmente denominam Custo (Cost) ao fator envolvendo "preço de venda" (low price), Qualidade (Quality-of-Conformance, Quality-of-Design) ao fator envolvendo as variáveis "projeto de produto" (making design changes in the product as desired by customer) e "qualidade na manufatura" (ensuring accuracy in manufacturing; ensuring consistency in manufacturing), além de Pontualidade (Dependability) ao fator envolvendo "pontualidade nas entregas" (meeting delivery dates).

A consistência interna dos respondentes foi verificada através da determinação do Alfa de Cronbach, o qual baseia-se na média das correlações estimadas entre um determinado fator e os todos os demais fatores. $\mathrm{O}$ limite padrão para o Alfa de Cronbach, a partir dos quais os dados são considerados confiáveis para análise estatística posterior, está definido em 0,55 (Mitchell e Jolley, 1996). Os Alfas de Cronbach estimados para cada fator estão listados na última linha da Tabela 3; todos os valores satisfazem o requisito padrão de consistência interna.

O agrupamento dos objetivos na Tabela 2 permitiu rotular os fatores, seguindo a lógica do modelo do cone de areia, em Noble (1995); os rótulos, definidos qualitativamente e justificados anteriormente, estão apresentados na segunda linha da Tabela 3. O \% da variância explicada por cada fator, bem como o \% de variância acumulada também vêm apresentados na Tabela 3. Já que somente cinco fatores foram mantidos para análise posterior, não foi possível identificar um fator que correspondesse ao rótulo inovação (inovação corresponde à última prioridade no modelo de Noble, não considerada originalmente nos modelos de Nakane, 1986, ou Ferdows e De Meyer, 1990). Assim, a hipótese $\mathrm{H} 1$ foi modificada para validar a seguinte seqüência de prioridades: qualidade, pontualidade, velocidade de entregas, custo e flexibilidade.

Após algumas substituições, os valores originalmente observados para os objetivos em cada empresa amostrada puderam ser reescritos em termos de escores fatoriais. Assim, medições dos 9 objetivos apresentados pela empresa 1 , por exemplo, foram substituídos por 5 escores, um para cada fator retido na análise. Os escores resultantes não são diretamente comparáveis, o que pode ser visualizado inspecionando as cargas em cada fator. Para permitir uma comparação direta entre fatores, as cargas na Tabela 3 foram normalizadas.

Os escores normalizados obtidos para cada fator foram, na seqüência, comparados entre si, com o objetivo de verificar as hipóteses H1, H2 e H3, para o conjunto de dados em análise. Obtiveram-se escores médios para cada fator. Como cada fator é dominado por um ou mais objetivos, seus escores médios refletem o grau médio de prioridade atribuído aos objetivos pelas empresas que responderam aos questionários. Assim, mediante comparação direta dos escores médios é possível definir uma seqüência de prioridades competitivas. Todavia, para proceder tal análise, é necessário verificar se existem diferenças significativas entre as médias dos escores dos fatores; uma vez assegurado que os escores médios diferem significativamente entre si, as médias podem ser diretamente comparadas e a seqüência de prioridades competitivas estabelecida. 
entrega na base do modelo do cone de areia. Se estes fatores efetivamente levarem a uma melhoria no desempenho econômico das empresas, seria razoável esperar uma correlação positiva entre os fatores e os indicadores de desempenho econômico. Analisando os resultados na Tabela 5, é possível verificar que os fatores 2 e 3 apresentam correlação positiva com todos os indicadores, mas somente as correlações no fator 2 são significativamente diferentes de zero. Assim, não foi possível reunir evidência empírica para validar a hipótese H4.

Em H1, verificou-se que o desenvolvimento de competências nas empresas pesquisadas seguia uma determinada seqüência. Os resultados na Tabela 5 apresentam as competências atualmente priorizadas pelas empresas (ou seja, o nível em que as empresas pesquisadas encontram-se atualmente posicionadas dentro do modelo acumulativo, relativamente ao desenvolvimento de suas prioridades competitivas): elas correspondem àquelas significativamente correlacionadas com os indicadores de desempenho econômico. Uma leitura possível das informações na Tabela 5 apontaria para entrega e pontualidade como prioridades sendo atualmente desenvolvidas pelas empresas e vistas como aquelas de maior impacto sobre os índices de desempenho econômico; benefícios advindos do foco em qualidade já se encontram consolidados, ao passo que custo e flexibilidade são as prioridades a serem desenvolvidas no futuro.

\section{Discussão}

Nesta seção, comparam-se os resultados apresentados na seção 5 com aqueles obtidos em estudos onde seqüências de prioridades de manufatura foram testadas; tais estudos incluem os trabalhos de Ferdows e De Meyer (1990), Roth e Miller (1992), Noble (1995) e Kim e Kim (1999). As análises estatísticas utilizadas nestes trabalhos são brevemente apresentadas e resultados obtidos são então comparados. O objetivo é identificar pontos fortes e fracos no procedimento analítico utilizado no presente trabalho e a aplicabilidade dos resultados aqui obtidos, à luz de estudos similares já consolidados.

O modelo de prioridades acumulativas em Ferdows e De Meyer (1990) apresenta qualidade na base, seguida por custo, flexibilidade e entrega. Esses autores usam os resultados na edição de 1988 do European Manufacturing Futures Project. Oito índices de desempenho diretamente relacionados às quatro prioridades acima são considerados (ao contrário de nossa abordagem, construtos não são derivados a partir dos índices). As empresas analisadas são classificadas como out-performers (com desempenho acima da média) ou under-performers (com desempenho claramente abaixo da média), com base nos escores apresentados nos índices medidos. Os autores tentam, então, identificar um padrão nos programas de melhorias adotados pelas empresas que valide o modelo acumulativo proposto. Desta forma, programas baseados em melhorias na qualidade deveriam afetar (positivamente) todos os índices de desempenho, já que a prioridade qualidade encontrase posicionada na base do modelo. Em contra-partida, programas que focam em melhorias nas entregas deveriam afetar somente aqueles índices de desempenho diretamente relacionados a entregas. Os autores identificam, através de inspeção visual, dois programas baseados em melhorias na qualidade que caracterizam empresas do tipo out-performers; a mesma investigação, todavia, não é conduzida enfocando programas de melhorias relacionados a prioridades posicionadas nos níveis mais altos do modelo acumulativos de prioridades.

O objetivo principal no estudo em Roth e Miller (1992) é verificar se o desempenho no negócio depende de fatores relacionados à prioridades de manufatura; a base de dados utilizada nesta análise é a mesma em Ferdows e De Meyer (1990). As empresas analisadas são primeiramente classificadas como leaders (líderes) ou laggers (seguidores), baseadas em seus escores em cinco índices de desempenho gerenciais, e então como weaklings, middlemen ou superstars, baseadas em seus escores em cinco índices de prioridades de manufatura (qualidade, entrega, escopo de mercado, flexibilidade e preço). Os autores utilizam índices obtidos diretamente dos questionários, e não construtos, em sua análise; a consistência interna das respostas utilizadas não é testada estatisticamente. No estudo, observou-se que empresas laggers apresentavam escores significativamente menores em todos os índices de desempenho gerenciais e de prioridades de manufatura. Apesar de sugerirem uma seqüência para as prioridades de manufatura (isto é, qualidade, entrega, escopo de mercado, flexibilidade e preço), os autores não a testam estatisticamente, ao contrário do que é apresentado no presente artigo. O foco da análise no trabalho de Roth e Miller (1992) é verificar se um bom desempenho global no negócio é função de boas práticas gerenciais e prioridades de manufatura bem desenvolvidas; esta é precisamente a proposta apresentada no presente artigo através da hipótese H4. Neste contexto, a seqüência de prioridades acumulativas aparece como um resultado secundário na análise feita por esses autores.

Noble (1995) procura validar um modelo acumulativo de prioridades competitivas usando dados de empresas de manufatura européias, coreanas e norteamericanas. O modelo hipotetizado pela autora apresenta qualidade na base, seguida por pontualida- 
de, entrega, custo, flexibilidade e inovação. Prioridades são avaliadas junto aos respondentes através de uma série de itens, agrupados pela autora para gerar construtos; o construto custo, por exemplo, é constituído de oito itens; já o construto entrega reúne apenas dois itens. É importante ressaltar que no procedimento apresentado no presente artigo, os construtos não são definidos pelo analista, mas obtidos através da estrutura de correlações dos itens. Os construtos em Noble (1995), formado por números distintos de itens, não são diretamente comparáveis. Desta forma, o modelo acumulativo é testado regredindo um construto sobre os demais, numa abordagem que utiliza regressão linear múltipla; coeficientes de regressão positivos evidenciariam a existência de uma seqüência de prioridades, com coeficientes maiores associados a prioridades na base do modelo. Os resultados apresentados pela autora não são conclusivos, já que registra-se a ocorrência de coeficientes de regressão que diferem nos sinais e na magnitude. Além disso, a tentativa de validação do modelo acumulativo através de análise de regressão apresentada por Noble (1995) é questionável, já que os construtos utilizados como variáveis independentes na análise apresentam severamente correlacionados, o que implica em multicolineariedade entre variáveis [ver Montgomery e Peck (1992), p. 189]. Nesta situação, os valores de significância dos coeficientes de regressão não são confiáveis.

O estudo em Kim e Kim (1999) utiliza a porção coreana da base de dados em Ferdows e De Meyer (1990) e Roth e Miller (1992). O objetivo do estudo é identificar as principais prioridades estratégicas e planos de ação adotados pela indústria coreana; a identificação da seqüência de prioridades de manufatura desenvolvidas por aquela indústria aparece como resultado secundário na análise apresentada. Os autores apresentam rankings de prioridades estratégicas, taxas de melhorias em índices de desempenho da manufatura e objetivos para a manufatura, baseados em seus escores observados. As diferenças entre os itens no ranking não são testadas estatisticamente quanto à sua significância. Os resultados limitam-se a ressaltar, de forma qualitativa, caminhos para a melhoria adotados pela indústria coreana.

A análise estatística apresentada no presente artigo pode ser comparada em complexidade com a análise em Noble (1995), apesar de levar a resultados mais confiáveis. A abordagem de natureza exclusivamente estatística adotada no presente artigo para definir os construtos de prioridades evita tendências eventualmente introduzidas pelo analista, o que pode ter sido o caso na análise em Noble (1995), por exemplo, dada a sua natureza. A análise aqui apresentada difere das demais expostas nos parágrafos acima por ter sido concebida especificamente para testar as hipóteses de pesquisa estabelecidas na seção 3 , levando a resultados conclusivos em todas as hipóteses levantadas.

\section{Conclusão}

Neste artigo, busca-se evidência empírica do modelo acumulativo de prioridades competitivas. O modelo acumulativo de prioridades foi originalmente proposto por Nakane (1986), sendo posteriormente desenvolvido por autores como Ferdows e De Meyer (1990) e Roth e Miller (1992). O modelo oferece um caminho para o desenvolvimento de uma série de prioridades competitivas, onde desenvolvimentos em prioridades a jusante auxiliariam desenvolvimentos em prioridades a montante. O modelo, assim, apresenta-se como uma alternativa à visão estática dos trade-offs entre prioridades competitivas.

Neste trabalho, aplicaram-se duas técnicas estatísticas, análise fatorial e análise de correlação, sobre dados obtidos do survey IMSS-II, com o objetivo de testar o modelo acumulativo de prioridades. Mais especificamente, foram testados três postulados básicos do modelo acumulativo: (i) a seqüência sugerida para o desenvolvimento de prioridades competitivas (testamos a seqüência proposta por Noble, 1995), (ii) qualidade como a primeira prioridade a ser desenvolvida e (iii) o impacto positivo do modelo sobre o desempenho econômico da firma. No que se refere a (i), em particular, este estudo é o primeiro a testar a validade da seqüência de desenvolvimento de prioridades competitivas através de análise estatística formal.

Os resultados forneceram evidência empírica de uma seqüência de prioridades competitivas, dada por qualidade, entrega, custo/pontualidade e flexibilidade. Esta seqüência corresponde, parcialmente, a seqüências determinadas em estudos anteriores, como Nakane (1986), Hall (1987) e Noble (1995). Os resultados também apontaram qualidade como a mais importante das prioridades competitivas, posicionando-a na base do modelo do cone de areia; tais resultados estão de acordo com aqueles em Ferdows e De Meyer (1988) e White (1996). Não pode-se identificar, todavia, a existência de correlação significativa entre a adoção do modelo acumulativo de prioridades e melhorias no desempenho econômico das empresas. A exata compreensão da relação entre a adoção do modelo acumulativo e o desempenho econômico em empresas será, assim, objeto de investigações futuras.

Os resultados deste trabalho apresentam três implicações práticas principais. Primeiro, o modelo acumulativo sugere que qualidade de processos tem um impacto não somente externo (enquanto objetivo competitivo da empresa) mas também interno, como suporte ao desenvolvimento de outras capacidades do 
sistema operativo. Isto sugere que gerentes de manufatura devem melhorar continuamente a qualidade de seus produtos e processos, independentemente dos níveis de qualidade efetivamente demandados em seus mercados. Segundo, os resultados suportam a idéia de que flexibilidade de operações deve ser desenvolvida somente após a obtenção de bons níveis de qualidade, entrega, velocidade e custo nas operações; parece difícil aumentar a variedade de produtos, processos e atividades executadas no sistema de operações sem que o sistema seja capaz de garantir níveis aceitáveis de desempenho nas outras áreas. Finalmente, dado o tempo e dificuldade envolvidos na obtenção de vantagens reais nas prioridades localizadas no topo do modelo (custo e flexibilidade), é fundamental que gerentes de operações tenham claramente definidos os limites para os quais ganhos nestas prioridades possam ser traduzidos em melhorias no desempenho global da empresa.

De um ponto de vista teórico, o estudo tem duas limitações principais que podem justificar o desenvolvimento de investigações futuras. Em primeiro lugar, este estudo teve como objetivo a validação teórica do modelo acumulativo, a partir de análise estatística de um survey de 283 empresas em 23 países. Apesar de demonstrar empiricamente a validade do modelo, o estudo não teve como objetivo "explicar” a maneira como as relações entre as prioridades competitivas se estabelecem na prática; por exemplo, como e porquê “qualidade” pode servir de base para o desenvolvimento das demais prioridades. Tal explicação pode ser fornecida por estudos qualitativos, especialmente análise de casos, buscando identificar as práticas mais adequadas para a implementação do modelo acumulativo em sistemas de manufatura. Em segundo lugar, como foi mencionado acima, o estudo não foi capaz de identificar a exata correlação entre a adoção do modelo acumulativo e melhorias no desempenho econômico das empresas. Estudos posteriores devem buscar estabelecer tal correlação, provavelmente utilizando um modelo de análise complementar ao utilizado neste estudo.

\section{Referências}

COLLINS, R.; CORDON, C.; JULIEN, D. An empirical test of the rigid flexibility model. $J$. Operations Management. Vol. 16, 1998, p. 133-146.

COLLINS, R.; SCHMENNER, R. Achieving rigid flexibility: factory focus for the 1990s. European Management Journal. Vol. 11, n. 4, 1993, p. 443-447.

DE MEYER, A. Manufacturing operations in Europe: where do we go next? European Management Journal. Vol. 16, n. 3, 1998, p. 262-271.
DE MEYER, A.; FERDOWS, K. Quality up, technology down: manufacturing improvement programs in Europe. Int. J. Technology Management, 1991, p. 136-158.

DE MEYER, A.; NAKANE, J.; MILLER, J.; FERDOWS, K. Flexibility: the next competitive battle - the manufacturing futures survey. Strategic Management Journal. Vol. 10, 1989, p. 135-144.

FERDOWS, K., DE MEYER, A. European manufacturers: the dangers of complacency. Columbia J. of World Business. Fall, 1988, p. 15-23.

FERDOWS, K.; DE MEYER, A. Lasting improvements in manufacturing: in search of a new theory. J. Operations Management. Vol. 9, n. 2, 1990, p. 168-184.

FERDOWS, K.; MILLER, J.; NAKANE, J., VOLLMANN, T. Evolving global manufacturing strategies: projections into the 1990s. Int. J. Oper. Prod. Manag. Vol. 6, n. 4, 1986, p. 6-16.

FLYNN, B; SCHROEDER, R.; FLYNN, E. World class manufacturing: an investigation of Hayes and Wheelwright's foundation. J. Operations Management. Vol. 17, 1999, p. 249-269.

HALL, R. Attaining Manufacturing Excellence. Homewood: Dow Jones-Irwin, 1987.

KIM, K.; KIM, T. Manufacturing strategies of Korean companies. Int. Studies of Management and Organization. Vol. 28, n. 4, 1999, p. 5-18.

MARDIA, K. Tests for univariate and multivariate normality. In Krishnaiah, P. (Ed.), Handbook of Statistic, Vol. 1: Analysis of Variance. Amsterdam, North Holland, 1980.

MITCHEL, M.; JOLLEY, J. Research Design Explained. New York: HBJ College, 1996.

MONTGOMERY, D.; RUNGER, G. Applied Statistics and Probability for Engineers. New York: John Wiley, 1994.

NAKANE, J. Manufacturing Futures Survey in Japan, A Comparative Survey 1983-1986. Tokio, System Science Institute, 1986.

NOBLE, M. Manufacturing strategy: testing the cumulative model in a multiple country context. Decision Sciences. Vol. 26, n. 5, 1995, p. 693-721.

NOBLE, M. Manufacturing competitive priorities and productivity: an empirical study. Int. J. Operations 
and Production Management. Vol. 17, n. 1, 1997, p. 85-99.

PORTH, S.; KATHURIA, R.; JOSHI, M. Performance impact of the fit between manufacturing priorities of general managers and manufacturing managers. Journal of Business and Economic Studies. Vol. 4, n. 1, 1998, p. 13-35.

RENCHER, A. Methods of Multivariate Analysis. New York: John Wiley, 1995.

ROTH, A.; MILLER, J. Success factors in manufacturing. Business Horizons, July, 1992, p.73-81.

SCHMENNER, R.; SWINK, M. On theory in operations management. J. Operations Management. Vol. 17, n. 1, 1998, p. 97-113.

SEBER, G. Multivariate Observations. New York: John Wiley, 1984.

SKINNER, W. The focused factory. Harvard Business Review. May-June, 1974, p. 113-121.

SPINA, G.; BARTEZZAGHI, E.; BERT, A.; CAGLIANO, R.; DRAAIJER, D.; BOER, H. Strategically flexible production: the multi-focused manufacturing paradigm. Int. J. Operations and Production Management. Vol. 16, n. 11, 1996, p. $20-$ 41.

WARD, P.; McCREERY, J.; RITZMAN, L.; SHARMA, D. Competitive priorities in operations management. Decision Sciences. Vol. 29, n. 4, 1998, p. $1035-1046$.

WHITE, G. A meta-analysis model of manufacturing capabilities. J. Operations Management. Vol. 14, n. 4, 1996, p. 315-331. 